\title{
Interaction between the Galaxies IC 2163 and NGC 2207
}

\section{Kaufman ${ }^{1}$, D. M. Elmegreen ${ }^{2}$, E. Brinks ${ }^{3}$, B. G. Elmegreen ${ }^{4}$, and}

M. Sundin ${ }^{5}$

${ }^{1}$ Department of Physics, Ohio State University, Columbus, U. S. A.

${ }^{2}$ Vassar College Observatory, Poughkeepsie, U. S. A.

${ }^{3}$ National Radio Astronomy Observatory, Socorro, U. S. A.

${ }^{4}$ IBM - Watson Research Center, Yorktown Heights, U. S. A.

${ }^{5}$ Department of Astronomy and Astrophysics, Chalmers University, Sweden

\section{ABSTRACT}

$V L A$ H I observations of the interacting pair IC 2163/NGC 2207 are presented. The velocity and structural anomalies of IC 2163 agree with predictions of $N$-body galaxy encounter simulations if IC 2163 recently underwent a strong, prograde, in-plane encounter with NGC 2207 . The velocity disturbances in NGC 2207 suggest that the main tidal force on NGC 2207 was perpendicular to the disk of NGC 2207.

\section{INTRODUCTION}

The spiral galaxies IC 2163 and NGC 2207, shown in Figure 1, are involved in a close tidal encounter. IC 2163 has an ocular shape (an eye-shaped central oval with a sharp apex at each end), intense star formation along the eyelid regions, and a double-parallel arm structure on the side opposite its companion, NGC 2207.

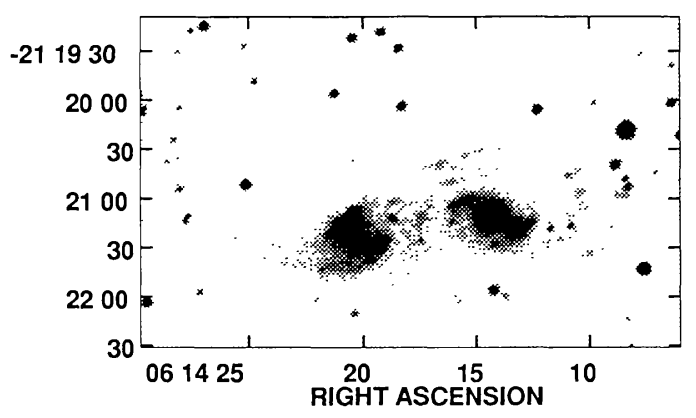

Fig. 1-R-band image taken by $\mathrm{H}$. Deeg of IC 2163 (left) and NGC 2207. The doubleparallel arm is on the eastern side of IC 2163 .

The optical morphology of IC 2163 is consistent with $N$-body simulations by Elmegreen et al. (1991) if IC 2163 underwent a strong, in-plane, prograde encounter with NGC 2207 during the last half-rotation. One component of the double arm is the usual tidal tail; the other component is produced by rapid streaming of tidally perturbed stars and gas from the companion (western) side of the galaxy. The simulations predict a velocity difference of 50 to $100 \mathrm{~km} \mathrm{~s}^{-1}$ between the two components of the double arm and large streaming motions along the oval. 
Using the $V L A$, we made $\mathrm{H}$ I observations of this galaxy pair to study the early stages of post-encounter evolution and to look for the velocity anomalies predicted by the numerical simulations.

\section{OBSERVED PROPERTIES OF IC 2163}

Our most useful VLA H d data on this system have an angular resolution of $13^{\prime \prime}(2 \mathrm{kpc})$ and a velocity resolution of $5 \mathrm{~km} \mathrm{~s}^{-1}$. We were able to separate the H I contributions of the two galaxies kinematically. Figure 2 shows the H I column density $\mathrm{N}(\mathrm{HI})$ associated with IC 2163. The central hole in H I coincides with the optical nucleus. In H I, tidal arms are located symmetrically on opposite sides of the nucleus, as predicted by the models (the western tidal arm is obscured optically by NGC 2207).

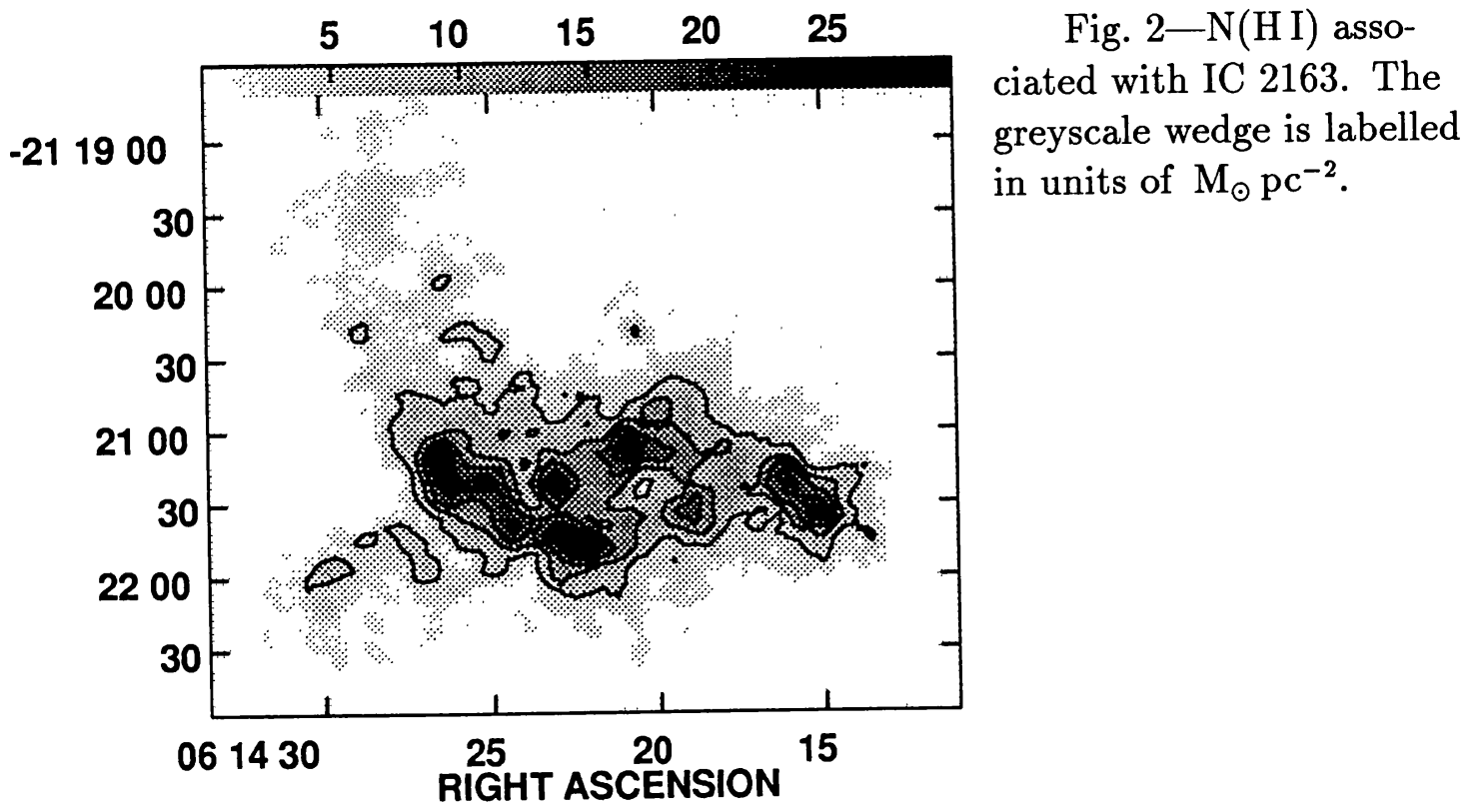

Since the two components of the eastern tidal arm in IC 2163 are separated mainly in declination, we made cuts across the double arm at fixed values of right ascension and displayed the $\mathrm{HI}$ emission in declination vs. velocity diagrams (see example in Fig. 3). We find two main velocity components on the double arm at each value of RA: the component with the higher velocity coincides with the northern component of the optical arm, while the other velocity component coincides with the southern component of the optical arm. The typical difference in the line-of-sight velocity between the two velocity components is $70-100 \mathrm{~km} \mathrm{~s}^{-1}$ and the separation is $20^{\prime \prime}-30^{\prime \prime}$. Thus two orbit streams occur on the tidal arm on the anti-collision side of the galaxy. The magnitude and sense of the velocity differences agree with the simulations in Elmegreen et al. (1991).

The kinematic minor axis of IC 2163 is nearly perpendicular to the apparent isophotal (optical and H I) minor axis of the oval. This unusual relative orientation 
of the axes is reproduced in our new $N$-body simulations and implies that the intrinsic shape of the disk is extremely oval. Also, from surface photometry in B and R-bands, we conclude that nearly all of the stars have been cleared out of the interarm regions of IC 2163 by the interaction and put into the inner oval and the tidal arms.

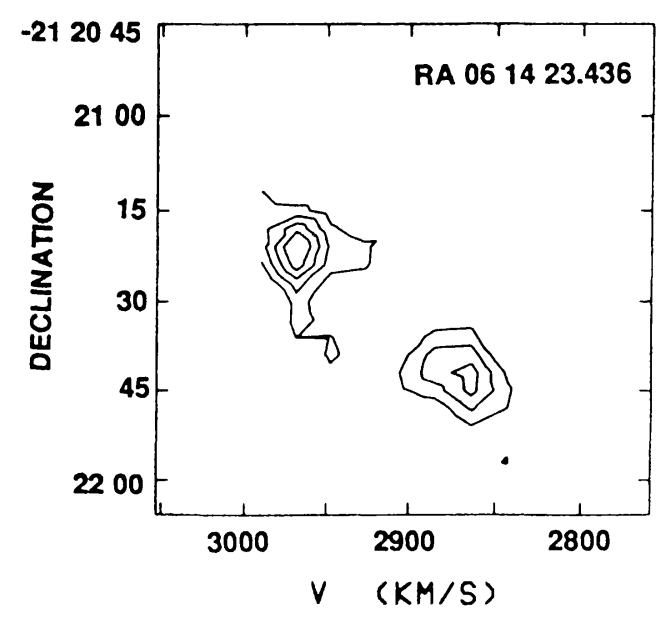

Fig. 3-The two components of the double arm in IC 2163: H I emission in a DEC vs. velocity display for a typical cut across the arm at fixed RA.

All of these observed properties of IC 2163 are also present in the $N$-body simulations. Thus our observations support the prograde, in-plane encounter model for IC 2163.

\section{OBSERVED PROPERTIES OF NGC 2207}

On short exposure optical images, such as Figure 1, NGC 2207 has a normal spiral pattern and a small, weak, central bar. Figure 4 shows the H I emission attributed to NGC 2207: the display on the left shows the H I column density image, and the display on the right shows the contours of the mean velocity field overlaid on the velocity dispersion image in greyscale. Both galaxies contain unusually massive H I clouds, comparable in mass to dwarf galaxies; the origin of these clouds as a consequence of the interaction is discussed by Elmegreen et al. (1993). The main H I emission from NGC 2207 forms a broad, clumpy ring that opens to the south and contains the optical spiral arms. However, on the eastern and western parts of the ring, the H I ridge-line often lies in the interarm region, between the stellar arms. In particular, an H I cloud with a mass of $10^{9} \mathrm{M}_{\odot}$ sits in the interarm region between the two stellar arms on the western part of the ring. The H I ring and the main optical disk are embedded in a large, low density H I puddle that extends nearly $3^{\prime}$ to the south and contains faint optical streamers. While the H I ring probably predates the present encounter, the present interaction has rearranged the distribution of gas in the ring and may have disrupted the southern part of the ring.

The velocity field (see Fig. 4) has a global S-shaped distortion. The gas in NGC 2207 is also very stirred up by the interaction; the H I line profiles in a large part of the main disk are broad and asymmetric. The velocity dispersion image in 
Fig. 4-The image on the left shows N(H I) associated with NGC 2207. The optical nucleus is in the $\mathrm{H}$ I hole, and the greyscale wedge is labelled in units of $\mathrm{M}_{\odot} \mathrm{pc}^{-2}$. The image on the right shows the velocity dispersion in NGC 2207 as greyscale with contours of the mean velocity field overlaid. The contour interval for the velocity field is $20 \mathrm{~km} \mathrm{~s}^{-1}$. The greyscale wedge for the velocity dispersion is labelled in units of $\mathrm{km} \mathrm{s}^{-1}$.

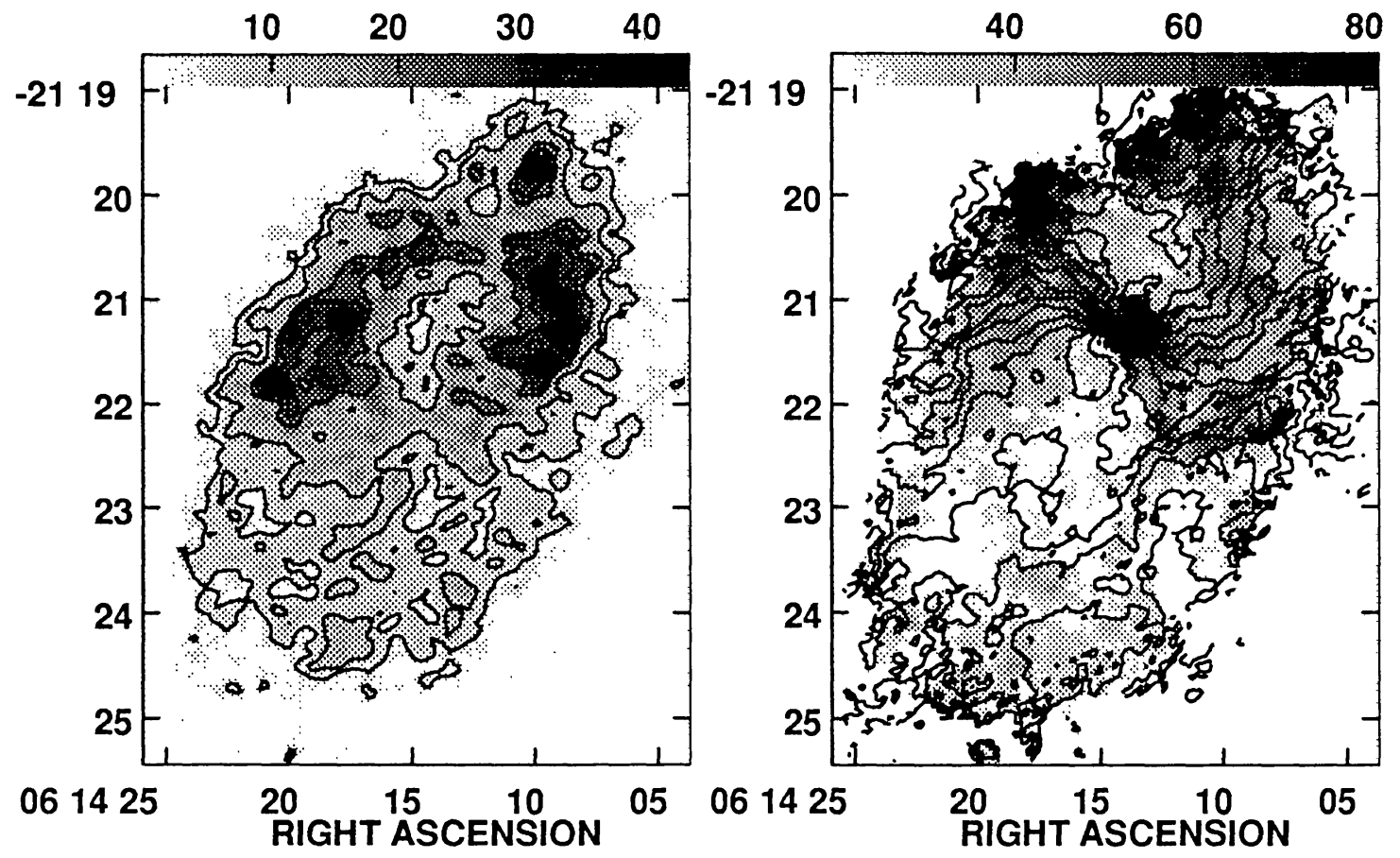

Figure 4 shows that the H I velocity dispersion is high $\left(40-60 \mathrm{~km} \mathrm{~s}^{-1}\right)$ in a large, butterfly-shaped region that covers much of the main disk and coincides with the $S$ shape of the velocity field. The anomalous velocities suggest that the disk is in the process of being warped by motions perpendicular to the disk, and thus the main tidal force on NGC 2207 was perpendicular to the plane.

The various disturbances in this system suggest that the collision line was nearly in the plane of IC 2163 but not in the plane of NGC 2207.

The National Radio Astronomy Observatory is operated by Associated Universities, Inc., under cooperative agreement with the National Science Foundation. This work was supported in part by NSF Grant AST-8914069 to M. K.

\section{REFERENCES}

Elmegreen, B. G., Kaufman, M., and Thomasson, M. 1993, Ap. J., 412, 90.

Elmegreen, D. M., Sundin, M., Elmegreen, B. G., and Sundelius, B. 1991, Astr. Ap., 244, 52 . 